\title{
LEFT INVERTIBILITY OF DISCRETE-TIME OUTPUT-QUANTIZED SYSTEMS: THE LINEAR CASE WITH FINITE INPUTS
}

\author{
NEVIO DUBBINI, BENEDETTO PICCOLI, AND ANTONIO BICCHI
}

\begin{abstract}
This paper studies left invertibility of discrete-time linear output-quantized systems. Quantized outputs are generated according to a given partition of the state-space, while inputs are sequences on a finite alphabet. Left invertibility, i.e. injectivity of I/O map, is reduced to left D-invertibility, under suitable conditions. While left invertibility takes into account membership to sets of a given partition, left D-invertibility considers only membership to a single set, and is much easier to detect. The condition under which left invertibility and left D-invertibility are equivalent is that the elements of the dynamic matrix of the system form an algebraically independent set. Our main result is a method to compute left D-invertibility (so also left invertibility for a full measure matrix set) for all linear systems with no eigenvalue of modulus one. Some examples are presented to show the application of the proposed method.
\end{abstract}

Left invertibility, uniform quantization, finite inputs, algebraic independent set, discrete time

\section{INTRODUCTION}

Left invertibility is an important problem of systems theory, which corresponds to injectivity of I/O map. It deals so with the possibility of recovering unknown inputs applied to the system from the knowledge of the outputs.

We investigate left invertibility of discrete-time linear output-quantized systems in a continuous state-space. In particular, inputs are arbitrary sequences of symbols in a finite alphabet: each symbol is associated to an action on the system. Information available on the system is represented by sequences of output values, generated by the system evolution according to a given partition of the state-space (quantization).

In recent years there has been a considerable amount of work on quantized control systems (see for instance [9], [19], [23] and references therein), stimulated also by the growing number of applications involving "networked" control systems, interconnected through channels of limited capacity (see e.g. [4, 7, 24, 25]). The quantization and the finite cardinality of the input set occur in many communication and control systems. Finite inputs arise because of the intrinsic nature of the actuator, or in presence of a logical supervisor, while output quantization may occur because of the digital nature of the sensor, or if data need a digital transmission.

Applications of left invertibility include fault detection in Supervisory Control and Data Acquisition (SCADA) systems, system identification, and cryptography ([11, 14]). Invertibility of linear systems is a well understood problem, first handled in [6], and then considered with algebraic approaches (see e.g. [21]), frequency domain techniques ([16], [17]), and geometric tools (cf. [18]). Invertibility of nonlinear systems is discussed in ([20]). More recent work has addressed the left invertibility for switched systems ([26]), and for quantized contractive systems ([10]).

The main intent of the paper is to show that the analysis of left invertibility can be substituted, under suitable conditions, by an analysis of a stronger notion, called left Dinvertibility. The condition under which left invertibility and left D-invertibility are equivalent is that the elements of the dynamic matrix of the system form an algebraically independent set (Theorem 5). Therefore the set of matrices for which left D-invertibility and 


\section{LEFT INVERTIBILITY OF DISCRETE-TIME OUTPUT-QUANTIZED SYSTEMS:} THE LINEAR CASE WITH FINITE INPUTS

left invertibility are equivalent is a full measure set. While left invertibility takes in account whether two states are in the same element of a given partition, left D-invertibility considers only the membership of a single state to a single set. For this reason left D-invertibility is much easier to detect. Our main result (Theorem 6) is a method to compute left Dinvertibility for all linear systems whose dynamic matrix has no eigenvalue of modulus one.

The main tools used in the paper are the theory of Iterated Function Systems (IFS), and a theorem of Kronecker. The use of IFS in relation with left invertibility is described in [10]. The Kronecker's theorem has to do with density in the unit cube of the fractional part of real numbers. By means of a particular construction illustrated in section 4 the problem of "turning" left D-invertibility into left invertibility can be handled with a Kronecker-type density theorem.

The paper is organized as follows. Section 2 is devoted to the definitions of left invertibility and uniform left invertibility. Section 3 illustrates the background knowledge. In section 4 the notion of D-invertibility is introduced and main results (Theorems 5 and 6 ) are proved. Section 5 contains a deeper study of unidimensional systems. In section 6 we present some examples and section 7 shows conclusions and future perspectives. The Appendix is devoted to technical proofs. Finally, in section 8, we collect all the notations used in the paper.

\section{BASIC SETTING}

Definition 1. The uniform partition of rate $\delta$ of $\mathbb{R}^{p}$ is

$$
\mathscr{P}=\bigcup_{i \in \mathbb{Z}^{p}} \mathscr{P}_{i}=\bigcup_{i_{1}, \ldots, i_{p} \in \mathbb{Z}}\left[i_{1} \delta,\left(i_{1}+1\right) \delta\left[\times \ldots \times\left[i_{p} \delta,\left(i_{p}+1\right) \delta[,\right.\right.\right.
$$

where $i=i_{1}, \ldots, i_{p} \cdot \diamond$

We consider systems of the form

$$
\left\{\begin{array}{l}
x(k+1)=A x(k)+B u(k) \\
y(k)=q(C x(k))
\end{array}\right.
$$

where $A \in \mathbb{R}^{d \times d}, B \in \mathbb{R}^{m \times d}, C \in \mathbb{R}^{p \times d}, x(k) \in \mathbb{R}^{d}$ is the state, $y(k) \in \mathbb{Z}^{p}$ is the output, and $u(k) \in \mathscr{U} \subset \mathbb{R}^{m}$ is the input. The map $q: \mathbb{R}^{p} \rightarrow \mathbb{Z}^{p}$ is induced by the uniform partition $\mathscr{P}=\bigcup_{i \in \mathbb{Z}^{p}} \mathscr{P}_{i}$ of $\mathbb{R}^{p}$ of rate $\delta$ through $q:\left(x \in \mathscr{P}_{i}\right) \mapsto i$ and will be referred to as the output quantizer. We assume that $\mathscr{U}$ is a finite set of cardinality $n$.

Remark 1. Without loss of generality in the system (1) we can suppose $\delta=1$ and $C=\pi_{p}$, the canonical projection on the first p coordinates.

Proof: We can indeed change the basis of $\mathbb{R}^{p}$ to achieve $\delta=1$, and the basis of $\mathbb{R}^{d}$ to achieve $C=\pi_{p} \cdot \diamond$

So we consider only systems of the form

$$
\left\{\begin{array}{l}
x(k+1)=A x(k)+B u(k)=f_{u(k)}(x(k)) \\
y(k)=\left\lfloor\pi_{p} x(k)\right\rfloor
\end{array}\right.
$$

where the floor function $\lfloor\cdot\rfloor$ is intended to work componentwise.

Definition 2. A pair of input strings $\{u(i)\}_{i \in \mathbb{N}},\left\{u^{\prime}(i)\right\}_{i \in \mathbb{N}}$ is uniformly distinguishable in $k$ steps (or with distinguishability time $k$ ) if there exists $l \in \mathbb{N}$ such that $\forall x(0), x^{\prime}(0) \in \mathbb{R}^{d}$ and $\forall m>l$ the following holds for the correspondent orbits:

$$
u(m) \neq u^{\prime}(m) \Rightarrow[y(m+1), \ldots, y(m+k)] \neq\left[y^{\prime}(m+1), \ldots, y^{\prime}(m+k)\right],
$$

(outputs $y(i)$ are referred to the system with initial condition $x(0)$ and inputs $u(i)$, while outputs $y^{\prime}(i)$ are referred to the system with initial condition $x^{\prime}(0)$ and inputs $\left.u^{\prime}(i)\right)$. In this case, we say that the strings are uniformly distinguishable with waiting time $l . \diamond$ 
Definition 3. A system of type (2) is uniformly left invertible (ULI) in $k$ steps if every pair of distinct input sequences is uniformly distinguishable in $k$ steps after a finite time $l$, where $k$ and $l$ are constant. $\diamond$

For a ULI system, it is possible to recover the input string until instant $m$ observing the output string until instant $m+k$. For applications, it is important to obtain an algorithm to reconstruct the input symbol used at time $m>l$ by processing the output symbols from time $m$ to $m+k$.

Notations: We indicate with $e_{i}$ the $i$-th vector of the canonical basis of $\mathbb{R}^{d}$, with $\left\langle v_{1}, \ldots, v_{i}\right\rangle$ the linear subspace generated by the vectors $v_{1}, \ldots, v_{i} \in \mathbb{R}^{d} . \diamond$

Definition 4. Define the quantization-diagonal set relative to the system (2) to be

$$
\begin{aligned}
Q & =\bigcup_{i_{1}, \ldots i_{p} \in \mathbb{Z}} \underbrace{\left\{\left[i_{1}, i_{1}+1\left[\times \ldots\left[i_{p}, i_{p}+1\left[\times\left\langle e_{p+1}, \ldots, e_{d}\right\rangle\right\}\right.\right.\right.\right.}_{\subset\left\langle e_{1}, \ldots, e_{d}\right\rangle} \times \\
& \times \underbrace{\left\{\left[i_{1}, i_{1}+1\left[\times \ldots\left[i_{p}, i_{p}+1\left[\times\left\langle e_{d+p+1}, \ldots, e_{2 d}\right\rangle\right\}\right.\right.\right.\right.}_{\subset\left\langle e_{d+1}, \ldots, e_{2 d}\right\rangle} \subset \mathbb{R}^{2 d}
\end{aligned}
$$

i.e. $Q$ contains all pairs of states that are in the same element of the partition $\mathscr{P} . \diamond$

To address invertibility, we are interested in the following system on $\mathbb{R}^{2 d}$ :

Definition 5. Define the doubled system relative to the system (2) to be

$$
\begin{gathered}
X(k+1)=F_{U(k)}(X(k))=\left[\begin{array}{c}
f_{u(k)}\left(x_{1}(k)\right) \\
f_{u^{\prime}(k)}\left(x_{2}(k)\right)
\end{array}\right]=\left[\begin{array}{c}
A x_{1}(k)+B u(k) \\
A x_{2}(k)+B u^{\prime}(k)
\end{array}\right] \\
\text { where } X(k)=\left(\begin{array}{c}
x_{1}(k) \\
x_{2}(k)
\end{array}\right), U(k)=\left(\begin{array}{c}
u(k) \\
u^{\prime}(k)
\end{array}\right) \cdot \diamond
\end{gathered}
$$

If there exists sequences $\{u(k)\},\left\{u^{\prime}(k)\right\}$, and an initial state in $Q$ such that the corresponding orbit of (3) remains in $Q$, then the two strings of inputs generate the same output for the system (2). So conditions ensuring that the state is outside $Q$ for some $k$ will be investigated to guarantee left invertibility.

\section{BACKGROUND: ITERATED FUnCTION Systems}

In this section we recall some results about Iterated Function System theory (see [3, 12] for general theory about IFS), in connection with the notions of invertibility of our interest.

\subsection{Contractive IFS theory.}

Definition 6. Let $(\mathbb{X}, d)$ be a complete metric space. A map $F: \mathbb{X} \rightarrow \mathbb{X}$ is contractive if $\exists c \in \mathbb{R}, 0<c<1$ such that $d(F(x), F(y)) \leq c d(x, y)$ for all $x, y \in \mathbb{X}$. A map $F: \mathbb{X} \rightarrow \mathbb{X}$ is expansive if $\exists c>1$ such that $d(F(x), F(y)) \geq c d(x, y)$ for all $x, y \in \mathbb{X}$. $\diamond$

Example 1. A linear map is contractive if its associated matrix has norm less than 1, where the norm of a matrix $A$ is defined by $\|A\|=\sup _{x \in \mathbb{R}^{d}} \frac{\|A x\|}{\|x\|}$. A linear map is expansive if for its associated matrix holds $\inf _{x \in \mathbb{R}^{d}} \frac{\|A x\|}{\|x\|}>1 . \diamond$

Definition 7. An Iterated Function System (IFS) is a collection $\left\{\mathbb{X}, F_{1}, \ldots, F_{n}\right\}$, where $(\mathbb{X}, d)$ is a complete metric space, $F_{i}: \mathbb{X} \rightarrow \mathbb{X}$ for $i=1, \ldots, n . \diamond$

Definition 8. Given an IFS, define $\mathfrak{F}_{t}=\left\{\left\{F_{i_{1}} \circ \ldots \circ F_{i_{t}}\right\}: i_{1}, \ldots, i_{t} \in\{1, \ldots, n\}\right\}$. The IFS is joint contractive if there exists $t \in \mathbb{N}$ such that all elements of $\mathfrak{F}_{t}$ are contractions. The IFS is joint expansive if there exists $t \in \mathbb{N}$ such that all elements of $\mathfrak{F}_{t}$ are expansive. $\diamond$ 


\section{LEFT INVERTIBILITY OF DISCRETE-TIME OUTPUT-QUANTIZED SYSTEMS: THE LINEAR CASE WITH FINITE INPUTS}

Example 2. An output-quantized linear system of type (2) is joint contractive if and only if for every eigenvalue $\lambda$ of the matrix $A$ it holds $|\lambda|<1$. It is joint expansive if and only for every eigenvalue $\lambda$ of the matrix $A$ it holds $|\lambda|>1 . \diamond$

Definition 9. A set $\mathscr{A} \subset \mathbb{X}$ is an attractor if for all initial condition $x(0) \in \mathbb{X}$ and for all $u \in \mathscr{U}^{\mathbb{N}} \lim _{k \rightarrow \infty} \operatorname{dist}(x(k), \mathscr{A})=0 . \diamond$

Definition 10. A set $\mathscr{I} \subset \mathbb{X}$ is an invariant set for an IFS if $\mathscr{I}=\bigcup_{i \in\{1, \ldots, n\}} F_{i}(\mathscr{I}) . \diamond$

Theorem 1. [3, 13] Let an IFS be joint contractive. Then, for every $u \in \mathscr{U}^{\mathbb{N}}$ the limit $\phi(u)=\lim _{k \rightarrow \infty} F_{u(1)} \circ \ldots \circ F_{u(k)}(x)$ exists for every $x \in \mathbb{X}$ and is independent of $x$. The set $\phi\left(\mathscr{U}^{\mathbb{N}}\right)=\mathscr{A}$ is the unique compact attractor and invariant set with respect to the IFS. $\diamond$

Definition 11. An address of a point $a \in \mathscr{A}$ is any member of the set $\phi^{-1}(a)=\left\{u \in \mathscr{U}^{\mathbb{N}}\right.$ : $\phi(u)=a\}$. The attractor is said to be totally disconnected if each point possesses a unique address. $\diamond$

3.2. Attractors and left invertibility. Thanks to Theorem 1, given a joint contractive system of type (2), for the doubled-system (3) it is possible to describe a set $\mathscr{A}$ that is both an attractor and an invariant set. Define $\Delta=\{(1,1),(2,2), \ldots,(n, n)\}$.

Definition 12. The graph $G_{k}$ associated to the attractor $\mathscr{A}$ is given by:

- The set of vertices $V=\left\{\mathscr{A}_{U(1) \ldots U(k)}=F_{U(k)} \circ \ldots \circ F_{U(1)}(\mathscr{A}): U(i) \in\{\mathscr{U} \times \mathscr{U}\}\right\}$.

- There is an edge from $\mathscr{A}_{U(1) \ldots U(k)}$ to $\mathscr{A}_{U^{\prime}(1) \ldots U^{\prime}(k)}$ if and only if $U(i+1)=U^{\prime}(i)$, for $i=1, \ldots, k-1$. In this case we say that the edge is induced by the input $U^{\prime}(k) . \diamond$

Definition 13. Consider the graph $G_{k}$, and delete all vertices (together with all starting and arriving edges) $\mathscr{A}_{U(1) \ldots U(k)}$ such that $\mathscr{A}_{U(1) \ldots U(k)} \cap\left\{\mathbb{R}^{2 d} \backslash Q\right\} \neq \emptyset$. This new graph is called internal invertibility graph, and denoted with $I G_{k}$. The set of vertices of $I G_{k}$ is denoted by $V_{I G_{k}}$.

Consider the graph $G_{k}$, and delete all vertices (together with all starting and arriving edges) $\mathscr{A}_{U(1) \ldots U(k)}$ such that $\mathscr{A}_{U(1) \ldots U(k)} \cap Q=\emptyset$. This new graph is called external invertibility graph, and denoted by $E G_{k}$. The set of vertices of $E G_{k}$ is denoted by $V_{E G_{k}} . \diamond$

Definition 14. A path $\left\{V_{i}\right\}_{i=0}^{\infty}$ on $E G_{k}$ or $I G_{k}$ is called proper path if the first edge is induced by an input not in $\Delta$. $\diamond$

Theorem 2. [10] Denote with $\partial Q$ the boundary of $Q$. Suppose that $\mathscr{A} \cap \partial Q=\emptyset$. Then there exists a (computable) $k$ such that $V_{I G_{k}}=V_{I G_{k}} \cap Q$, and the system (2) is ULI if and only if $I G_{k}$ does not contain arbitrary long proper paths. $\diamond$

3.3. IFS techniques for joint expansive systems. If the system (2) is joint expansive, then $f_{u}(\cdot)$ admit an inverse for every $u \in \mathscr{U}$. Therefore it is possible to define a correspondent system on $\mathbb{R}^{2 d}$ :

Definition 15. The inverse-doubled system, relative to the system (2), is

$$
Z(k+1)=G_{U(k)}(Z(k))=\left[\begin{array}{c}
f_{u(k)}^{-1}\left(z_{1}(k)\right) \\
f_{u^{\prime}(k)}^{-1}\left(z_{2}(k)\right)
\end{array}\right]
$$

where $Z(k)=\left(\begin{array}{c}z_{1}(k) \\ z_{2}(k)\end{array}\right) ; U(k)=\left(\begin{array}{c}u(k) \\ u^{\prime}(k)\end{array}\right) \cdot \diamond$

The system (4) gives rise to an attractor $\mathscr{R} \subset \mathbb{R}^{2 d}$, since it is joint contractive: the attractor $\mathscr{R}$ can be described also as the set of initial conditions that can start a bounded orbit of the doubled system (3):

Theorem 3. [10] Suppose that the system [2] is joint expansive. If $\{X(k)\}$ is an infinite bounded orbit of the doubled system (3), then $\forall k \in \mathbb{N} X(k) \in \mathscr{R}$. Consequently, if we restrict to bounded orbits, Theorem 2 applies to the attractor $\mathscr{R} . \diamond$ 


\section{DIFFERENCE SYSTEM AND D-INVERTIBILITY}

Notations: We indicate with $\operatorname{frac}(\cdot): \mathbb{R} \rightarrow \mathbb{Z}$ the function that associates to each real number its fractional part:

$$
\operatorname{frac}(r)=r-\lfloor r\rfloor
$$

Moreover we define $\pi_{i}: \mathbb{R}^{d} \rightarrow \mathbb{R}^{i}$ to be the orthogonal projection on the first $i$ coordinate axes, while $\varpi_{i}: \mathbb{R}^{d} \rightarrow \mathbb{R}$ is the orthogonal projection on the $i$-th coordinate axis. $\diamond$

Definition 16. The difference system associated with the system (2) is

$$
z(k+1)=A z(k)+B v(k)=f_{v(k)}(z(k))
$$

where $z(k) \in \mathbb{R}^{d}, v(k) \in \mathscr{V}=\mathscr{U}-\mathscr{U}=\left\{u-u^{\prime}: u \in \mathscr{U}, u^{\prime} \in \mathscr{U}\right\} . \diamond$

Remark 2. The difference system represents at any instant the difference between the two states $z(k)=x(k)-x^{\prime}(k)$ when the input symbols $u(k)-u^{\prime}(k)=v(k)$ are performed. So we are interested in understanding the conditions under which

$$
\{z(k)\} \cap\{]-1,1[\}^{p} \times\left\langle e_{p+1}, \ldots, e_{d}\right\rangle=\emptyset .
$$

Indeed, this implies that $y(k) \neq y^{\prime}(k)$. The converse is obviously not true. $\diamond$

Definition 17. Consider the difference system. If $z(0)$ is an initial condition and $\left(v(1), \ldots, v\left(k_{2}\right)\right)$ a sequence of inputs of the difference system, we let $D_{k_{1}}^{k_{2}}\left(z(0), v(1), \ldots, v\left(k_{2}\right)\right)$ denote the sequence $\left(\pi_{p} z\left(k_{1}\right), \ldots, \pi_{p} z\left(k_{2}\right)\right)$ generated by the difference system (5) with initial condition $z(0)$ and input string $\left(v(1), \ldots, v\left(k_{2}\right)\right) . \diamond$

Definition 18. A pair of input strings $\{u(i)\}_{i \in \mathbb{N}},\left\{u^{\prime}(i)\right\}_{i \in \mathbb{N}}$ is uniformly D-distinguishable in $k$ steps (or with distinguishability time $k$ ) if there exists $l \in \mathbb{N}$ such that $\forall x(0), x^{\prime}(0) \in \mathbb{R}^{d}$ and $\forall m>l$ the following holds:

$$
v(m) \neq 0 \Rightarrow D_{m+1}^{m+k}(z(0), v(1), \ldots, v(m+k)) \notin \underbrace{]-1,1\left[{ }^{p} \times \ldots \times\right]-1,1\left[^{p}\right.}_{k \text { times }},
$$

where $z(0)=x(0)-x^{\prime}(0)$ and $v(i)=u(i)-u^{\prime}(i)$. In this case, we say that the strings are uniformly D-distinguishable with waiting time $l . \diamond$

Definition 19. A system of type (2) is uniformly left D-invertible (ULDI) in $k$ steps if every pair of distinct input sequences is uniformly $D$-distinguishable in $k$ steps after a finite time $l$, where $k$ and $l$ are constant. $\diamond$

Remark 3. Thanks to Remark 2 uniform left D-invertibility implies uniform left invertibility. $\diamond$

We are going to prove the first main theorem, that is based on a density theorem of Kronecker.

Definition 20. The numbers $\vartheta_{1}, \ldots, \vartheta_{M} \in \mathbb{R}$ are linearly independent over $\mathbb{Z}$ if the following holds:

$$
k_{1}, \ldots, k_{M} \in \mathbb{Z}: k_{1} \vartheta_{1}+\ldots,+k_{M} \vartheta_{M}=0 \Rightarrow k_{1}=\ldots=k_{M}=0 . \diamond
$$

Theorem 4 (Kronecker). [15] If $\vartheta_{1}, \ldots, \vartheta_{M}, 1 \in \mathbb{R}$ are linearly independent over $\mathbb{Z}$, then, for every $\alpha_{1}, \ldots, \alpha_{M} \in \mathbb{R}$ the set of points

$$
\left\{\left[\operatorname{frac}\left(\alpha_{1}+l \vartheta_{1}\right), \ldots, \operatorname{frac}\left(\alpha_{M}+l \vartheta_{M}\right)\right]: l \in \mathbb{R}\right\}
$$

is dense in the unit cube of $\mathbb{R}^{M}$.

Considering the difference system (Definition 16, we are interested in orbits completely included in

$$
(]-1,1[)^{p} \times\left\langle e_{p+1}, \ldots, e_{d}\right\rangle .
$$

The following proposition shows that under a very weak condition orbits completely included in (]$-1,1[)^{p} \times\left\langle e_{p+1}, \ldots, e_{d}\right\rangle$ must be bounded. 
Proposition 1. Suppose that the matrix A does not have an eigenvector belonging to $\left\langle e_{p+1}, \ldots, e_{d}\right\rangle$. Then there exists a bounded set I such that, if $\{z(k)\}_{k \in \mathbb{N}} \subset(]-1,1[)^{p} \times$ $\left\langle e_{p+1}, \ldots, e_{d}\right\rangle$ is an orbit of the difference system, then $\{z(k)\}_{k \in \mathbb{N}} \subset I . \diamond$

Proof: See Appendix. $\diamond$

Remark 4. The set of matrices $A \in \mathbb{R}^{d^{2}}$ that have an eigenvector in $\left\langle e_{p+1}, \ldots, e_{d}\right\rangle$ is a zero measure set. $\diamond$

Define now $\mathbb{S}_{D}(B, \mathscr{U})$ to be the set of matrices $A \in \mathbb{R}^{d^{2}}$ such that the system 2. is uniformly left D-invertible. Define $\mathbb{S}(B, \mathscr{U})$ to be the set of matrices $A \in \mathbb{R}^{d^{2}}$ such that the system (2) uniformly left invertible.

Definition 21. Indicate with $\mathbb{Q}\left[\zeta_{1}, \ldots, \zeta_{N}\right]$ the ring of polynomials in the variables $\zeta_{i}$ with coefficients in $\mathbb{Q}$. The set of numbers $\alpha_{1}, \ldots, \alpha_{N} \in \mathbb{C}$ is said to be algebraically independent if

$$
0 \neq p\left(\zeta_{1}, \ldots, \zeta_{N}\right) \in \mathbb{Q}\left[\zeta_{1}, \ldots, \zeta_{N}\right] \Rightarrow p\left(\alpha_{1}, \ldots, \alpha_{N}\right) \neq 0 . \diamond
$$

Theorem 5. Suppose that in the d-dimensional system (2) the set of elements of the matrix $A$ is algebraically independent. Then the system is uniformly D-invertible if and only if it is uniformly left invertible.

This in turn implies that $\mathbb{S}(B, \mathscr{U}) \backslash \mathbb{S}_{D}(B, \mathscr{U})$ has measure zero in $\mathbb{R}^{d^{2}}$ for every $B, \mathscr{U}$.

Proof: See appendix. $\diamond$

4.1. D-invertibility of output-quantized linear systems. We are going to show how to detect left D-invertibility of any linear systems without eigenvalues of modulus one. Suppose that, if $\lambda$ is an eigenvalue of the matrix $A$, then $|\lambda| \neq 1$. Denote with $E_{c}, E_{e}$ respectively the contractive and the expansive eigenspaces of the matrix $A$. Because of the hypothesis on the eigenvalues we have $E_{c}+E_{e}=\left\{x+y: x \in E_{c}, y \in E_{e}\right\}=\mathbb{R}^{d}$.

Now consider the following two systems respectively on $E_{c}, E_{e}$, that are joint contractive:

$$
\begin{aligned}
& z_{c}(k+1)=f_{v(k)}\left(z_{c}(k)\right) ; \\
& z_{e}(k+1)=f_{v(k)}^{-1}\left(z_{e}(k)\right) ;
\end{aligned}
$$

where $z_{c} \in E_{c}, z_{e} \in E_{e}, v(k) \in \mathscr{V}$. The above systems must have invariant attractors $\mathscr{T}^{c}, \mathscr{T}^{e}$. Moreover the set $\mathscr{T}^{e}$ is formed of all initial conditions giving rise to bounded orbits of system (Theorem 3 )

$$
x_{e}(k+1)=f_{v(k)}\left(x_{e}(k)\right),
$$

here $x_{e} \in E_{e}$. Let us denote with $\mathscr{T}$ the attractor

$$
\mathscr{T}=\mathscr{T}^{c}+\mathscr{T}^{e}=\left\{x+y: x \in \mathscr{T}^{c}, y \in \mathscr{T}^{e}\right\} .
$$

We can now apply the construction of the external and internal invertibility graphs (Definition 13 for the attractor $\mathscr{T}$, substituting $Q$ with \{]$-1,1[\}^{p} \times\left\langle e_{p+1}, \ldots, e_{d}\right\rangle$, and calling a path $\left\{V_{1}, \ldots, V_{i}\right\}$ on $E G_{k}$ or $I G_{k}$ proper if it is induced by an input $v \in \mathscr{V}, v \neq 0$. Denoting with $\partial\left((]-1,1[)^{p} \times\left\langle e_{p+1}, \ldots, e_{d}\right\rangle\right)$ the boundary of (]$-1,1[)^{p} \times\left\langle e_{p+1}, \ldots, e_{d}\right\rangle$ we have the following

Theorem 6. Suppose that, if $\lambda$ is an eigenvalue of the matrix $A$, then $|\lambda| \neq 1$, that $\mathscr{T} \cap \partial\left((]-1,1[)^{p} \times\left\langle e_{p+1}, \ldots, e_{d}\right\rangle\right)=\emptyset$, and that $A$ does not have an eigenvector in $\left\langle e_{p+1}, \ldots, e_{d}\right\rangle$. Let $k$ be such that $I G_{k}=E G_{k}$. Then the system (2) is uniformly left $D$ invertible if and only if $I G_{k}$ does not contain arbitrary long proper paths. 
Proof: Since $A$ does not have an eigenvector in $\left\langle e_{p+1}, \ldots, e_{d}\right\rangle$, by Proposition 1 all orbits of the difference system included in (]$-1,1[)^{p} \times\left\langle e_{p+1}, \ldots, e_{d}\right\rangle$ must be bounded, and, by Theorem 3 must be included in the attractor $\mathscr{T}$. By Theorem 2 the system is uniformly D-invertible if and only if $I G_{k}$ does not contain arbitrary long proper paths. $\diamond$

Remark 5. Theorem 6 gives an explicit, algorithmically implementable, way to compute ULDI of a system. By Theorem 5 we are able to compute ULI in the same way for systems with a full measure set of matrices $A$ (the conditions on the eigenvalues do not affect the full measure). $\diamond$

\section{OUTPUT-QUANTIZED LINEAR SYSTEMS OF DIMENSION 1}

Linear systems of dimension 1 assume the following form, deriving from (2):

$$
\left\{\begin{array}{l}
x(k+1)=a x(k)+u(k) \\
y(k)=\lfloor x(k)\rfloor
\end{array}\right.
$$

This is a contractive system if $|a|<1$ and an expansive system if $|a|>1$. If $|a|<1$ the invertibility problem can be solved with the methods of section 3 (see [10]).

The next Theorem shows a necessary condition for the ULI of a system of type (9): if it is not satisfied we construct inductively a pair of strings that gives rise to the same output.

Theorem 7. Suppose that in the system (9) $|a|>2$. If there exist $u_{1}, u_{2} \in \mathscr{U}, u_{1} \neq u_{2}$ such that $\left|u_{1}-u_{2}\right|<a$, then the system is not ULI.

Proof: We will consider sequences of sets of type

$$
\left\{\begin{array}{l}
S_{i+1}=\left\{a\left(S_{i}\right)+u(i)\right\} \bigcap\left\{a\left(S_{i}\right)+u^{\prime}(i)\right\} \bigcap \mathscr{P}(i+1) \\
S_{0}=[0,1[,
\end{array}\right.
$$

where $u(i), u^{\prime}(i) \in\left\{u_{1}, u_{2}\right\}$ and $\mathscr{P}(i+1) \in \mathscr{P}$ is chosen at each step to maximize the measure of $S_{i+1}$.

In the sequence 10 take $u(1)=u_{1}, u^{\prime}(1)=u_{2}$ and $\mathscr{P}(1)$. Since $\left|u_{1}-u_{2}\right|<a$, there exists a $\mathscr{P}(1) \in \mathscr{P}$ such that $\mu\left(S_{1}\right)>0$. Then, for $i>1$ define

$$
u(i)=u^{\prime}(i)=u_{1} \text {. }
$$

Since $|a|>2$ there exists an $i_{0}$ such that $\mu\left(S_{i_{0}}\right)=1$, therefore, applying again $u\left(i_{0}+1\right)=$ $u_{1}$ and $u^{\prime}\left(i_{0}+1\right)=u_{2}$

$$
\mu\left\{A\left(S_{i_{0}}\right)+B u_{1} \cap A\left(S_{i_{0}}\right)+B u_{2}\right\}>0 .
$$

So there exists $x_{0}, x_{0}^{\prime} \in \mathbb{R}$ and $\left(u(1), \ldots, u\left(i_{0}+1\right)\right),\left(u^{\prime}(1), \ldots, u^{\prime}\left(i_{0}+1\right)\right)$, with $u(1) \neq u^{\prime}(1)$ and $u\left(i_{0}+1\right) \neq u^{\prime}\left(i_{0}+1\right)$, such that for the corresponding outputs it holds

$$
\left(y(0), \ldots, y\left(i_{0}+1\right)\right)=\left(y^{\prime}(0), \ldots, y^{\prime}\left(i_{0}+1\right)\right)
$$

It is then enough to point out that, since we can achieve every pair of states $x, x^{\prime} \in S_{i_{0}}$ in the above described way, we can again go on in the same way and find a new instant $i_{1}$, a pair of initial states $x_{1,0}, x_{1,0}^{\prime}$, and control sequences $\left(u(1), \ldots, u\left(i_{1}\right)\right),\left(u^{\prime}(1), \ldots, u^{\prime}\left(i_{1}\right)\right)$, with $u\left(i_{1}\right) \neq u^{\prime}\left(i_{1}\right)$, such that for the corresponding output it holds

$$
\left(y(0), \ldots, y\left(i_{1}\right)\right)=\left(y^{\prime}(0), \ldots, y^{\prime}\left(i_{1}\right)\right) .
$$

Finally, we can achieve by induction an increasing finite sequence, but arbitrarily long, of instants $i_{k}$, pairs of initial states $\left(x_{k, 0}, x_{k, 0}^{\prime}\right)$, and sequences of controls $\left(u(1), \ldots, u\left(i_{k}\right)\right),\left(u^{\prime}(1), \ldots, u^{\prime}\left(i_{k}\right)\right)$ with $u(i) \neq u^{\prime}(i)$ if $i=i_{j}+1$ for $j=1, \ldots, k-1$ such that such that for the corresponding output it holds

$$
\left(y(0), \ldots, y\left(i_{k}\right)\right)=\left(y^{\prime}(0), \ldots, y^{\prime}\left(i_{k}\right)\right) .
$$

This contradicts the uniform left invertibility property. $\diamond$ 


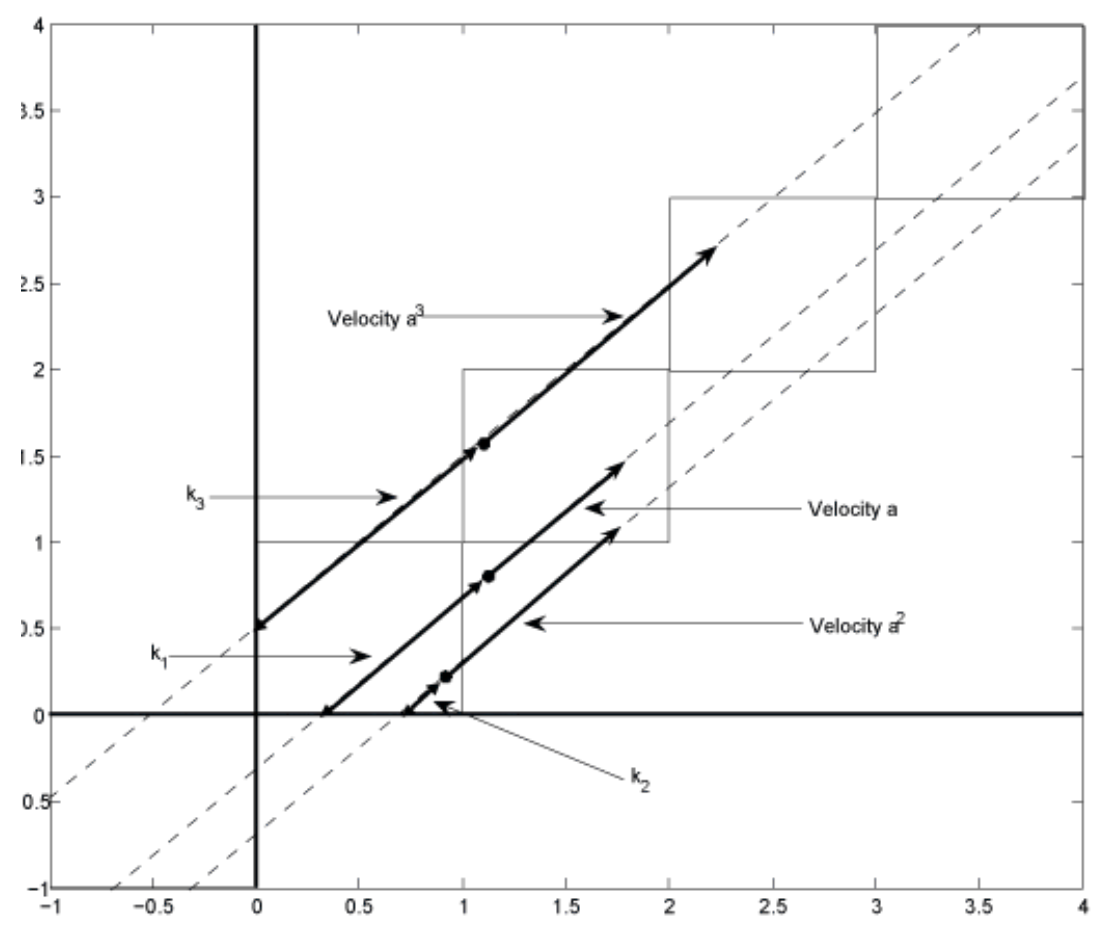

FIGURE 1 . Here, for $i=1,2,3$, the point $X_{t}(i)$ has a distance $k_{i}$ from the union of positive coordinate axes along the line $r_{i}$ (drawn with a dashed line), and "velocity" $a^{i}$ (with respect to $t$ )

We simplify notations to handle the unidimensional ULI problem. Consider the correspondent doubled-system of dimension 2 given by (3), and suppose that there exists at least one proper orbit included in the set

$$
Q^{\prime}=\left\{\left(\begin{array}{c}
t \\
t
\end{array}\right)+\left(\begin{array}{c}
s \\
0
\end{array}\right): s \in\right]-1,1[, t \in \mathbb{R}\} .
$$

Take as initial condition $X_{t}(0)=\left(\begin{array}{c}t \\ t\end{array}\right)+\left(\begin{array}{c}s \\ 0\end{array}\right) \in \mathbb{R}^{2}$, with $t$, considered as a parameter, varying in $\mathbb{R}$ and $s \in]-1,1[$ fixed. Then, for fixed input string

$$
X_{t}(k)=\left(\begin{array}{c}
a^{k} t+a^{k} s+a^{k-1} u_{1}+\ldots+u_{k} \\
a^{k} t+a^{k-1} u_{1}^{\prime}+\ldots+u_{k}^{\prime}
\end{array}\right)=a^{k}\left(\begin{array}{c}
t \\
t
\end{array}\right)+\left(\begin{array}{c}
c_{k} \\
c_{k}^{\prime}
\end{array}\right) .
$$

Suppose that an orbit $\left\{X_{t}(i)\right\}_{i=1}^{\infty}$ of the doubled-system is included in $Q^{\prime}$. We can see the points $X_{t}(i)$, when $t$ varies in $\mathbb{R}$, as points moving along the line

$$
r_{i}=\left\{a^{i}\left(\begin{array}{c}
t \\
t
\end{array}\right)+\left(\begin{array}{c}
c_{i} \\
c_{i}^{\prime}
\end{array}\right): t \in \mathbb{R}\right\}
$$

with initial condition $\left(\begin{array}{c}c_{i} \\ c_{i}^{\prime}\end{array}\right)$ and velocity $a^{i}$. Call $k_{i}$ the distance between the point $\left(\begin{array}{c}c_{i} \\ c_{i}^{\prime}\end{array}\right)$ and the union of positive coordinate axes along the line $r_{i}$ (refer to the Fig. 1). 
Definition 22. A number $\alpha \in \mathbb{C}$ is called algebraic if there exists a polynomial $p(x) \in \mathbb{Z}[x]$ such that $p(\alpha)=0$. In this case the minimum degree of a polynomial with such a property is called the degree of $\alpha$. A number $\alpha \in \mathbb{C}$ is called trascendental if it is not algebraic. $\diamond$

Proposition 2. Suppose that the unidimensional linear system (9) is not uniformly left $D$-invertible. If $a$ is an algebraic number of degree $m$ then the system is not uniformly invertible in $m-1$ steps.

Proof: Since the system is not uniformly left D-invertible there exist arbitrary long orbits of the doubled system included in $Q^{\prime}$. Fix one of these orbits of length greater than $m-1$.

If, for every $\varepsilon>0$, and every $k_{1}, \ldots, k_{m} \in \mathbb{R}$ there exists a $t \in \mathbb{R}$ such that

$$
\operatorname{frac}\left(k_{i}+\frac{a^{i}}{\sqrt{2}} t\right)<\varepsilon \text { for } i=0, \ldots, m-1
$$

then the system (9) is not uniformly left invertible in $m-1$ steps: it can be showed adapting the proof of Lemma 1 (see also Fig. 1). Equation (11) is equivalent to find integers $N_{0}, \ldots, N_{m}$ such that for every $i=0, \ldots, m$

$$
N_{i} \leq k_{i}+\frac{a^{i}}{\sqrt{2}} t<N_{i}+\varepsilon
$$

But, if $a$ is algebraic of degree $m$, then numbers $a^{i}, i=0, \ldots, m-1$ are linearly independent over $\mathbb{Z}$, and by Theorem 4 there always exists a $t$ such that equation (11) holds, and so the system is not uniformly invertible in $m-1$ steps. $\diamond$

The following Theorem can be deduced from Proposition 2, or directly from Theorem 5. observing that an algebraically independent set of one element is a trascendental number.

Theorem 8. Suppose that a is trascendental. Then the system (9) is uniformly invertible if and only if it is uniformly D-invertible. $\diamond$

Proposition 3. The unidimensional system (9) is either ULDI in time 1, or not ULDI at all.

Proof: A sufficient condition for uniform left D-invertibility in one step is

$$
\forall v \in \mathscr{V}, v \neq 0:|v| \geq|a|+1:
$$

indeed in this hypothesis $\forall v \in \mathscr{V}, v \neq 0$

$$
]-1,1[\cap\{a \cdot(]-1,1[)+v\}=]-1,1[\cap]-a+v, a+v[=\emptyset
$$

We now prove that if $\exists v \in \mathscr{V}, v \neq 0:|v|<|a|+1$, then the system is not uniformly left D-invertible. Indeed in this case the system

$$
\left\{\begin{array}{l}
a x_{1}+v=x_{2} \\
a x_{2}-v=x_{1}
\end{array}\right.
$$

has the solution $x_{1}=\frac{-v}{a+1}, x_{2}=\frac{v}{a+1}$. Since $\left|x_{1}\right|,\left|x_{2}\right|<1$ the difference system has the infinite orbit $\left\{x_{1}, x_{2}, x_{1}, x_{2}, \ldots\right\} \subset(]-1,1[)^{p} \times\left\langle e_{p+1}, \ldots, e_{d}\right\rangle$. Therefore system 9$\}$ is not left D-invertible. $\diamond$

Corollary 1. Consider the unidimensional system (9), with trascendental a. Then it is either ULI in one step, or it is not ULI. $\diamond$

Remark 6. It's easy to see that a system of the form (9) is uniformly D-invertible in one step if there for all $u_{1}, u_{2} \in \mathscr{U}$ it holds $\left|u_{1}-u_{2}\right|>a+1$. Therefore we have this summarizing situation for unidimensional systems:

- $|a|<1$ : ULI can be detected with methods described in section 3. 


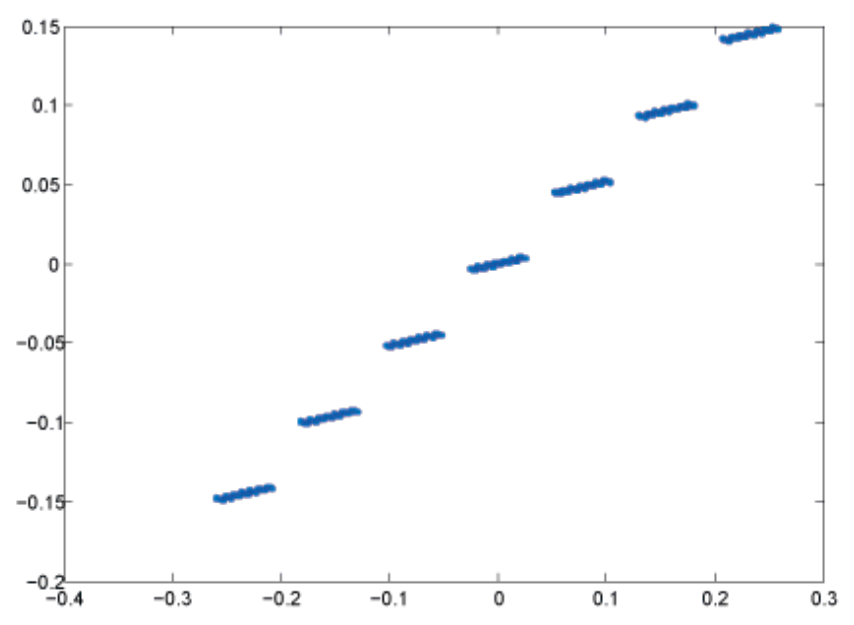

FIGURE 2. Attractor of system (13).

- $|a|>2: \begin{cases}\min _{u_{1}, u_{2} \in \mathscr{U}}\left|u_{1}-u_{2}\right|<|a|, & \text { the system is not ULI; } \\ \min _{u_{1}, u_{2} \in \mathscr{U}}\left|u_{1}-u_{2}\right|>|a|+1, & \text { the system is ULI in 1 step; } \\ |a| \leq \min _{u_{1}, u_{2} \in \mathscr{U}}\left|u_{1}-u_{2}\right| \leq|a|+1, & \star \text { holds. }\end{cases}$
- $1 \leq|a| \leq 2: \star$ holds.

$\star$ The unidimensional system (9) is either ULDI in time 1, or not ULDI at all. With the additional hypothesis of trascendence of a the system is ULI in one step or it is not ULI. $\diamond$

\section{EXAMPLES}

Consider the system (2). As stated in Theorem 5, if the elements of the matrix $A$ forms an algebraically independent set, then uniform left $\mathrm{D}$-invertibility is equivalent to uniform left invertibility. A standard method to construct algebraically independent sets can be easily deduced from the following Theorem of Lindemann and Weierstrass:

Theorem 9. [2] Suppose that the numbers $\alpha_{1}, \ldots, \alpha_{N}$ are linearly independent over $\mathbb{Q}$. Then $e^{\alpha_{1}}, \ldots, e^{\alpha_{N}}$ are an algebraically independent set. $\diamond$

Example 3. Consider the system (2) with

$$
A=\left(\begin{array}{ll}
e^{\sqrt{5}} & e^{\sqrt{3}} \\
e^{\sqrt{2}} & e^{\sqrt{7}}
\end{array}\right), B=\left(\begin{array}{c}
1 \\
1
\end{array}\right), \mathscr{U}=\{0, \pm 1,2\} \quad y(k)=\left\lfloor\pi_{1} x(k)\right\rfloor .
$$

The two eigenvalue of $A$ are approximately 6.3531 and 17.0974, so system (12) is joint expansive. The difference system is then joint expansive too. The inverse difference system is given by

$$
x(k+1)=A^{-1}(x(k)-v(k)), v(k) \in \mathscr{V}=\{0, \pm 1, \pm 2, \pm 3\}
$$

which is joint contractive. It is possible to show (see Fig. 2) that the attractor of the inverse difference system (13) is included in $]-1,1[\times \mathbb{R}$. So the system is not uniformly left D-invertible.

Moreover the elements of the matrix A, by Theorem 9 are an algebraically independent set because $\sqrt{5}, \sqrt{2}, \sqrt{3}, \sqrt{7}$ are linearly independent over $\mathbb{Q}$. So by Theorem 5 system (12) is not uniformly left-invertible. $\diamond$ 


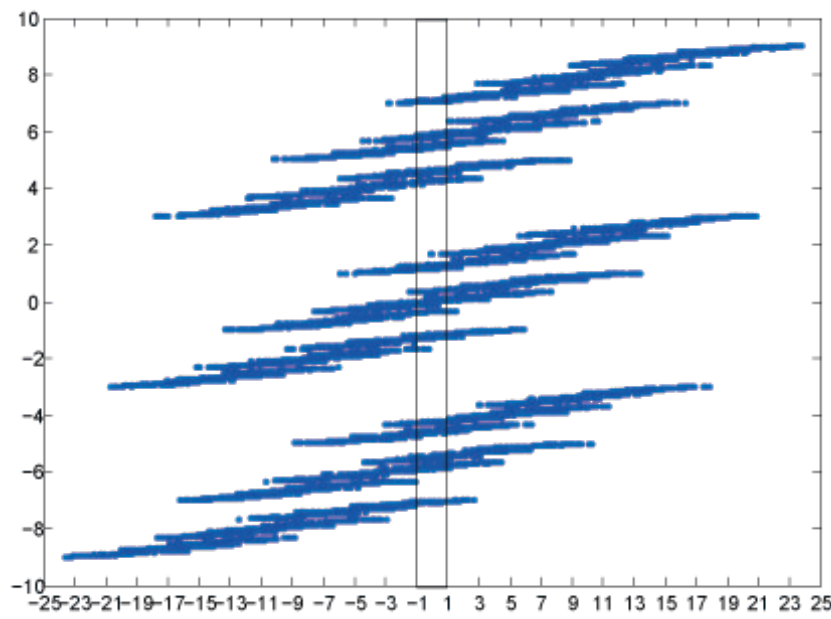

FIGURE 3. Attractor of the inverse difference system on $E_{c}$.

Example 4. Consider the system (2) with

$$
A=\left(\begin{array}{ccc}
2 & 0 & 0 \\
0 & \frac{1}{2} & 1 \\
0 & 0 & \frac{1}{3}
\end{array}\right), B=\left(\begin{array}{l}
2 \\
3 \\
6
\end{array}\right), \quad \mathscr{U}=\{0,1\} \quad y(k)=\left\lfloor\pi_{2} x(k)\right\rfloor .
$$

We have so $\mathscr{V}=\{0, \pm 1\}$. The three eigenvalue of the matrix are $\frac{1}{2}, \frac{1}{3}$ and 2 so we can apply Theorem 6. Therefore we split $\mathbb{R}^{3}$ in $E_{c}=\mathbb{R}^{2}$ (identified with $\{0\} \times \mathbb{R}^{2}$ ) and $E_{e}=\mathbb{R}$ (identified with $\mathbb{R} \times\{0\} \times\{0\}$ ). The attractor $\mathscr{A}_{e}$ relative to the inverse difference system on $E_{e}$ is $[-2,2]$, while the attractor $\mathscr{A}_{c}$ relative to the inverse difference system on $E_{c}$ is drawn in Fig. 3. We are interested in orbits of the inverse difference system on $E_{e}$ that remains in $]-1,1\left[\right.$, and in orbits of the difference system on $E_{c}$ that remains in $]-1,1[\times \mathbb{R}$. It's easy to see that

$$
\left(\begin{array}{cc}
\frac{1}{2} & 1 \\
0 & \frac{1}{3}
\end{array}\right)\left(\mathscr{A}_{c} \cap\right]-1,1[\times \mathbb{R})+\left(\begin{array}{l}
3 \\
6
\end{array}\right) \bigcap\left\{\mathscr{A}_{c} \cap\right]-1,1[\times \mathbb{R}\}=\emptyset
$$

so, no matter the behavior of the system on $E_{e}$, system $(14)$ is uniformly left D-invertible in one step, therefore uniformly invertible in one step. $\diamond$

The last example illustrates the difference between left D-invertibility and left invertibility.

Example 5. Consider the unidimensional system (9) with

$$
a=1 / 2, \quad \mathscr{U}=\{-1,0,1\} .
$$

We are going to show that system (15) is uniformly left invertible but not uniformly left D-invertible.

To show that the system is not ULDI consider the following orbit with initial condition $x_{0}=\frac{1}{2}$ :

$$
x_{k+1}= \begin{cases}\frac{1}{2} x(k+1), & x(k)<0 \\ \frac{1}{2} x(k-1), & x(k)>0 .\end{cases}
$$

Clearly $x(k) \in]-1,1[$ for every $k \in \mathbb{N}$, so system $(15)$ is not ULDI. 
Nonetheless system (15) is ULI in 1 step. Consider indeed the quantization-diagonal set (defined in Definition 4)

$$
Q=\bigcup_{i \in \mathbb{Z}}[i, i+1[\times[i, i+1[
$$

and observe that

$$
\frac{1}{2} Q+\left(\begin{array}{c}
u \\
u^{\prime}
\end{array}\right) \bigcap Q=\emptyset \forall u \neq u^{\prime}
$$

This in turn implies that system (15) is ULI in 1 step. $\diamond$

\section{Conclusions}

In this paper we studied left invertibility of output-quantized linear systems, and we proved that it is equivalent, under suitable conditions, to left D-invertibility, a stronger notion, much easier to detect (Theorem 6). More precisely the condition under which left invertibility and left D-invertibility are equivalent is that the elements of the dynamic matrix of the system form an algebraically independent set. Therefore the set of matrices for which left D-invertibility and left invertibility are equivalent is a full measure set (Theorem 5). Moreover there is a standard way to create matrices whose elements are an algebraically independent set (Theorem 9p. Notice that algebraic conditions play a central role in investigation of left invertibility of quantized systems as well in other fields when a quantization is introduced (see for instance [4, 8]).

Future research will include further investigation on the equivalence between left invertibility and left D-invertibility to matrices whose elements are not algebraically independent.

\section{APPENDIX}

Proof of Proposition 1 It's easy to see that the matrix $A$ does not have an eigenvector included in $\left\langle e_{p+1}, \ldots, e_{d}\right\rangle=\operatorname{Ker}\left(\pi_{p}\right)$ if and only if

$$
\bigcap_{i=0}^{d-p-1} A^{i}\left[\operatorname{Ker}\left(\pi_{p}\right)\right]=\{0\}
$$

Define $\mathscr{F}: \mathbb{R}^{d} \rightarrow \mathbb{R}^{d}$

$$
\mathscr{F}(x)=\bigcup_{v \in \mathscr{V}} A x+B v
$$

We show that $\mathscr{F}^{\circ}(d-p)\left((]-1,1[)^{p} \times\left\langle e_{p+1}, \ldots, e_{d}\right\rangle\right)$, where $\mathscr{F}^{\circ}(d-p)$ is the composition of $\mathscr{F}$ for $p$ times, is bounded: this is sufficient for the existence of the bounded set $I$.

(]$-1,1[)^{p} \times\left\langle e_{p+1}, \ldots, e_{d}\right\rangle$ is the convex space delimited by the $(d-p)$-dimensional affine subspaces

$$
\Psi_{i_{0}}^{j_{0}}=i_{0} e_{j_{0}}^{p} \times\left\langle e_{p+1}, \ldots, e_{d}\right\rangle \quad i_{0} \in\{-1,1\}, j_{0} \in[1, p],
$$

where $e_{j_{0}}^{p}$ is the $j_{0}$-th vector of the canonical basis of $\mathbb{R}^{p}$. So $z(1)$ belongs to the convex space delimited by the $(d-p-1)$-dimensional affine subspaces

$$
\Psi_{i_{0}, i_{1}}^{j_{0}, j_{1}}=A\left(i_{1} e_{j_{1}}^{p} \times\left\langle e_{p+1}, \ldots, e_{d}\right\rangle\right)+B v(1) \bigcap i_{0} e_{j_{0}}^{p} \times\left\langle e_{p+1}, \ldots, e_{d}\right\rangle .
$$

Therefore $z(d-p)$ belongs to the convex space delimited by the $(d-p-(d-p))=0$ dimensional affine subspaces (points)

$$
\Psi_{i_{0}, \ldots, i_{d-p}}^{j_{0}, \ldots, j_{d-p}}=i_{0} e_{j_{0}}^{p} \times\left\langle e_{p+1}, \ldots, e_{d}\right\rangle \cap\left\{\bigcap_{k=1}^{d-p} A^{k}\left(i_{k} e_{j_{k}}^{p} \times\left\langle e_{p+1}, \ldots, e_{d}\right\rangle\right)+B v(k)\right\} .
$$

By hypothesis $16, z(d-p)$ belongs so to the convex space delimited by a finite number of points, that is a bounded set, i.e. $I . \diamond$ 


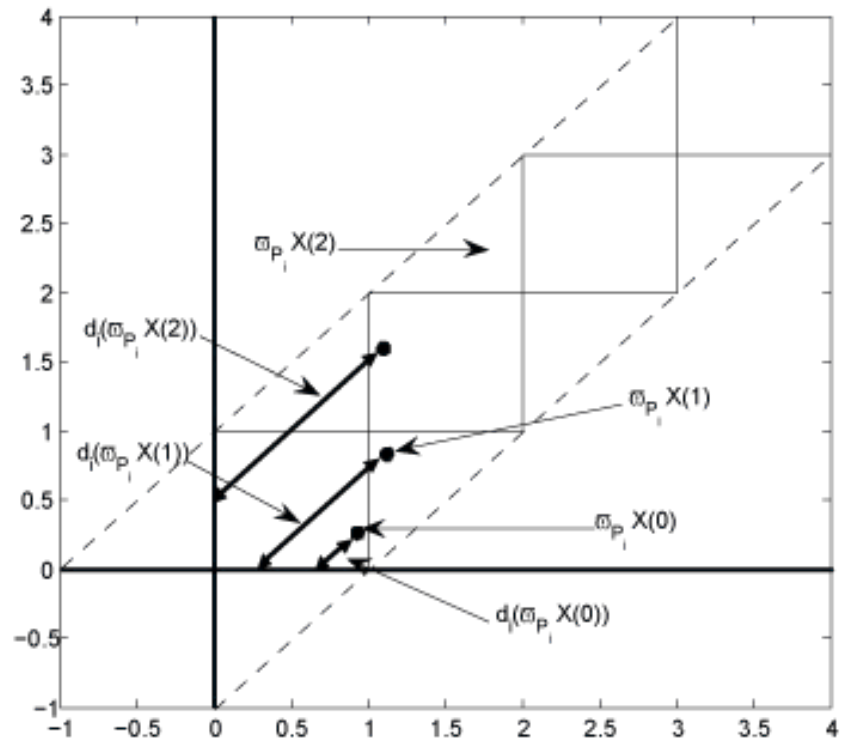

FIGURE 4. In this Figure the set $Q_{i}$ is represented by the squares on the diagonal, while the set $Q_{i}^{\prime}$ is the strip between the two dashed lines.

The following technical lemma gives a necessary condition for uniform left invertibility, a basilar ingredient in the proof of Theorem 5 Let us parametrize the possible pairs of states $\left(x, x^{\prime}\right) \in \mathbb{R}^{2 d}$ such that $x^{\prime}-x \in(]-1,1[)^{p} \times\left\langle e_{p+1}, \ldots, e_{d}\right\rangle$ with the set

$$
I_{0}=\left\{\left(t_{1}, \ldots, t_{d}, t_{1}+s_{1}, \ldots, t_{p}+s_{p}, \tilde{t}_{p+1}, \ldots, \tilde{t}_{d}\right)^{T}: t_{i}, \tilde{t}_{j} \in \mathbb{R}, s_{k} \in\right]-1,1[\} .
$$

\section{Proof of Theorem 5}

Definition 23. If $X=\left(t_{1}, \ldots, t_{d}, t_{1}+s_{1}, \ldots, t_{p}+s_{p}, \tilde{t}_{p+1}, \ldots, \tilde{t}_{d}\right)^{T} \in I_{0}$, for $i=1, \ldots, p$ define $d_{i}(X)$ to be the distance, measured along the line

$$
\{t_{1}, \ldots, \underbrace{\tau_{i}}_{\text {varies }}, \ldots, t_{d}, t_{1}+s_{1}, \ldots, \underbrace{\tau_{i}}_{\text {varies }}+s_{i}, \ldots, t_{p}+s_{p}, \tilde{t}_{p+1}, \ldots, \tilde{t}_{d}: \tau_{i} \in \mathbb{R}\}
$$

from the set

$$
\Omega_{i}=\left\{X \in \mathbb{R}^{2 d}: \varpi_{j} X=0 \text { for } j \neq i, i+d\right\} . \diamond
$$

Lemma 1. Fix a sequence $\{U(j)\}_{j \in \mathbb{N}}$ of inputs for the doubled system (3). Suppose that $\left.\forall \varepsilon>0, \forall m \in \mathbb{N}, \forall s_{1}, \ldots, s_{p} \in\right]-1,1\left[\right.$, there exists $t_{1}, \ldots, t_{d}, \tilde{t}_{p+1}, \ldots, \tilde{t}_{d} \in \mathbb{R}$ such that, if $\{X(j)\}_{j=0}^{m} \subset I_{0}$ is the orbit of the doubled system 3 with $X(0)=\left(t_{1}, \ldots, t_{d}, t_{1}+s_{1}, \ldots, t_{p}+\right.$ $\left.s_{p}, \tilde{t}_{p+1}, \ldots, \tilde{t}_{d}\right)$, the following holds

$$
\operatorname{frac}\left(\frac{d_{i}(X(j))}{\sqrt{2}}\right)<\varepsilon
$$

for every $i=1, \ldots, p, j=1, \ldots, m$. Then the system is not uniformly left invertible.

Proof: Suppose that an orbit $\{X(i)\}_{i=1}^{\infty}$ is included in $I_{0}$ and consider the 2-dimensional plane $\left\langle e_{i}, e_{d+i}\right\rangle$. Define

$$
\begin{gathered}
Q_{i}^{\prime}=\left\{(t, t+s) \in \mathbb{R}^{2}: t \in \mathbb{R}, s \in\right]-1,1[\} \subset\left\langle e_{i}, e_{d+i}\right\rangle, \\
Q_{i}=\bigcup_{j \in \mathbb{Z}}\left[j, j+1\left[\times\left[j, j+1\left[\subset\left\langle e_{i}, e_{d+i}\right\rangle .\right.\right.\right.\right.
\end{gathered}
$$


Now observe that $\operatorname{frac}\left(\frac{d_{i}(X(j))}{\sqrt{2}}\right)=0$ if and only if $X(j)$ belongs to some translation of $\Omega_{i}$, along the diagonal of the 2-dimensional plane $\left\langle e_{i}, e_{d+i}\right\rangle$, that is entirely included in $Q_{i}$, i.e. a translation that takes $\Omega_{i}$ to the "bottom-left boundary" of a square of $Q_{i}$. It's now easy to see that, for every $X \in \mathbb{R}^{2 d}$ there exists $\varepsilon>0$ such that, if $\operatorname{frac}\left(\frac{d_{i}(X)}{\sqrt{2}}\right)<\varepsilon$ then $X \in Q_{i}$. Therefore, if the relations (17) are satisfied, then there exists an arbitrary long orbit included in $Q$ (see also Fig. 4). $\diamond$

Proposition 4. Suppose that the entries of a matrix $A=\left\{a_{i j}\right\} \in \mathbb{R}^{d \times d}$ are an algebraically independent set, and denote with $a_{i j}^{(k)}$ the entries of the matrix $A^{k}$. Then the set

$$
\left\{a_{i j}^{(k)}: i, j=1, \ldots d ; k=1, \ldots, N\right\}
$$

is a linearly independent set for every $N \in \mathbb{N}$.

Proof: First, note that all $a_{i j}^{(k)}$ are polynomials of degree $k$ in the $a_{i j}$. Since the $a_{i j}$ are algebraically independent, they can be treated formally as the independent variables of polynomials in $d^{2}$ variables (more precisely there exists a ring isomorphism between $\mathbb{Q}\left[a_{11}, a_{12}, \ldots, a_{d d}\right]$ and the ring of polynomials in $d^{2}$ variables $\mathbb{Q}\left[x_{1}, \ldots, x_{d^{2}}\right]$, see [1]). If a nontrivial linear combination of the elements of the set $(18)$ is zero, then there exists a nontrivial polynomial in the $a_{i j}$ which is zero, so there exists a $k_{0}$ such that a nontrivial linear combination of the $a_{i j}^{\left(k_{0}\right)}$, s, seen as polynomials in the $a_{i j}$ 's, which is zero. These are the entries of the matrix $A^{k_{0}}$, so there would exist a nontrivial linear relation among these entries. Suppose this is the case. If this linear relation results in a linear relation among polynomials which is not identically zero, we are done. Indeed, if there exists $\lambda_{i j k_{0}} \in \mathbb{Q}$ and $x_{11}, \ldots, x_{d d} \in \mathbb{R}$ such that (note that the entries $a_{i j}^{\left(k_{0}\right)}$ of the matrix $A^{k_{0}}$ are seen as polynomials in the variables $a_{11}, \ldots, a_{d d}$, renamed as $x_{11}, \ldots, x_{d d}$ )

$$
\sum_{i, j} \lambda_{i j k_{0}} a_{i j}^{\left(k_{0}\right)}\left(x_{11}, \ldots, x_{d d}\right) \neq 0,
$$

then, substituting the $a_{i j}$ 's to the $x_{i j}$ 's, it is not possible that

$$
\sum_{i, j} \lambda_{i j k_{0}} a_{i j}^{\left(k_{0}\right)}\left(a_{11}, \ldots, a_{d d}\right) \neq 0
$$

since the $a_{i j}$ 's are algebraically independent.

Therefore we only have to show that it is not possible that

$$
\sum_{i, j} \lambda_{i j k_{0}} a_{i j}^{\left(k_{0}\right)}\left(x_{11}, \ldots, x_{d d}\right) \equiv 0
$$

i.e. that this polynomial cannot be identically zero. Now note that the matrices $M=$ $\left\{m_{i j}\right\} \in \mathbb{R}^{d \times d}$ whose entries do not satisfy the (nontrivial) linear relation $\sum_{i, j} \lambda_{i j k_{0}} m_{i j}=0$ form a full measure set, dense in $\mathbb{R}^{d \times d}$. On the other hand also the matrices with distinct eigenvalues form a full measure set, dense in $\mathbb{R}^{d \times d}$. Therefore there exists a matrix $\bar{M}=\left\{\bar{m}_{i j}\right\}$ having distinct eigenvalues, whose entries do not satisfy the linear relation $\sum_{i, j} \lambda_{i j k_{0}} \bar{m}_{i j}=0$.

Since $\bar{M}$ has distinct eigenvalues, there exists a matrix $B$ such that $B^{k_{0}}=\bar{M}$ (diagonalize and take $k_{0}$-roots of the eigenvalue). Denote with $b_{i j}^{\left(k_{0}\right)}$ the entries of the matrix $B^{k_{0}}$ : then the $b_{i j}^{\left(k_{0}\right)}$,s do not satisfy the linear relation $\sum_{i, j} \lambda_{i j k_{0}} b_{i j}^{\left(k_{0}\right)}=0$, since $B^{k_{0}}=\bar{M}$. This implies that $\sum_{i, j} \lambda_{i j k_{0}} a_{i j}^{\left(k_{0}\right)}\left(x_{11}, \ldots, x_{d d}\right)$ is not identically zero as a polynomial. $\diamond$

We now prove Theorem 5. Consider the state $X(m)$ of the doubled-system 3 ) at instant $m$ given by an initial condition $X(0) \in I_{0}$ and an input sequence 


$$
U(1)=\left(\begin{array}{c}
u(1) \\
u^{\prime}(1)
\end{array}\right), \ldots, U(m)=\left(\begin{array}{c}
u(m) \\
u^{\prime}(m)
\end{array}\right) \in \mathscr{U} \times \mathscr{U}:
$$

Then

$$
X(m)=\left(\begin{array}{cc}
A & 0 \\
0 & A
\end{array}\right)^{m} X_{0}+\ldots+\left(\begin{array}{c}
B u(m) \\
B u^{\prime}(m)
\end{array}\right)
$$

Suppose that the ( $d$-dimensional) system 2 is not uniformly left D-invertible. So there exists arbitrarily long orbits of the ( $2 d$-dimensional) doubled-system (3) included in $I_{0}$. In the following we provide conditions such that $\left.\forall \varepsilon>0, \forall m \in \mathbb{N}, \forall s_{1}, \ldots, s_{p} \in\right]-1,1$, there exists $t_{1}, \ldots, t_{d}, \tilde{t}_{p+1}, \ldots, \tilde{t}_{d} \in \mathbb{R}$ such that, if $\{X(j)\}_{j=0}^{m}$ is the orbit of the doubledsystem 3 with $X(0)=\left(t_{1}, \ldots, t_{d}, t_{1}+s_{1}, \ldots, t_{p}+s_{p}, \tilde{t}_{p+1}, \ldots, \tilde{t}_{d}\right)$, then the following holds

$$
\operatorname{frac}\left(\frac{d_{i}(X(j))}{\sqrt{2}}\right)<\varepsilon,
$$

for every $i=1, \ldots, p, j=1, \ldots, m$. Therefore the system will be not uniformly left invertible by Lemma 1. These conditions will be verified by a full measure set. Consider the set

$$
\mathbb{S}^{\prime}=\left\{A \in \mathbb{R}^{d \times d}:\left\{a_{i j}\right\}_{i, j=1}^{d} \text { is an algebraically independent set }\right\} .
$$

Set $A \in \mathbb{S}^{\prime}$. For $i=1, \ldots, p, \varpi_{\left\langle e_{i}, e_{d+i}\right\rangle} X(j)$ has the form

$$
\begin{aligned}
& \left(\begin{array}{c}
\varpi_{i} X(j) \\
\varpi_{i+d} X(j)
\end{array}\right)=\varpi_{\langle i, i+d\rangle}\left[\left(\begin{array}{cc}
A & 0 \\
0 & A
\end{array}\right)^{j} X(0)+\left(\begin{array}{cc}
A & 0 \\
0 & A
\end{array}\right)^{j-1} U(1)+\ldots+U(j)\right]= \\
& =\varpi_{\langle i, i+d\rangle}\left[\left(\begin{array}{cccccc}
a_{11} & \ldots & a_{1 d} & 0 & \ldots & 0 \\
\vdots & \ddots & \vdots & \vdots & \ddots & \vdots \\
a_{d 1} & \ldots & a_{d d} & 0 & \ldots & 0 \\
0 & \ldots & 0 & a_{11} & \ldots & a_{1 d} \\
\vdots & \ddots & \vdots & \vdots & \ddots & \vdots \\
0 & \ldots & 0 & a_{d 1} & \ldots & a_{d d}
\end{array}\right) \quad\left(\begin{array}{c}
t_{1} \\
\vdots \\
t_{d} \\
t_{1}+s_{1} \\
\vdots \\
t_{p}+s_{p} \\
\tilde{t}_{p+1} \\
\vdots \\
\tilde{t}_{d}
\end{array}\right)+\text { const. }\right]= \\
& =\left(\begin{array}{l}
c_{i, 1}^{(j)} t_{1}+\ldots+c_{i, d}^{(j)} t_{d} \\
c_{i, 1}^{(j)} t_{1}+\ldots+c_{i, p}^{(j)} t_{p}+c_{i, p+1}^{(j)} \tilde{t}_{p+1}+\ldots+c_{i, d}^{(j)} \tilde{t}_{d}
\end{array}\right)+\text { const. }
\end{aligned}
$$

where $c_{i, l}^{(j)}$ is the entry $(i, l)$ of the matrix $A^{j}$. By Proposition 4 the set $\left\{a_{i l}^{(j)}: i, l=1, \ldots d ; j=1, \ldots N\right\}$ is a linearly independent set, so, by Kronecker's Theorem (Theorem 4) there exists a choice of $\left(t_{1} \ldots, t_{d}, \tilde{t}_{p+1}, \ldots, \tilde{t}_{d}\right)$ such that equation 17$)$ is satisfied, and Lemma 1 thus apply.

To prove that the set of matrices with algebraically independent entries are a full measure set, first observe that the set of polynomial $P \in \mathbb{Q}\left[\zeta_{1}, \ldots, \zeta_{d^{2}}\right]$ is countable. For a single polynomial $P$ the set

$$
0_{P}=\left\{\left(x_{1}, \ldots, x_{d^{2}}\right) \in \mathbb{R}^{d^{2}}: P\left(x_{1}, \ldots, x_{d^{2}}\right)=0\right\}
$$

is a finite union of manifolds of dimension at most $d^{2}-1$. So the measure of $0_{P}$ is zero. Moreover

$$
\mathbb{S}^{\prime}=\bigcup_{P \in \mathbb{Q}\left[\zeta_{1}, \ldots, \zeta_{d^{2}}\right]} 0_{P}
$$


i.e. $\mathbb{S}^{\prime}$ is a countable union of sets of measure zero, which in turn implies that the measure of $\mathbb{S}^{\prime}$ is zero. $\diamond$

\section{NOTATIONS}

The authors made an effort to simplify notations, though they are intrisically complex. For this reason in this "special" section we collect the notations used in this paper, ordered as their appearance.

- $\mathscr{P}=\bigcup \mathscr{P}_{i}$ is the uniform partition. Without loss of generality the rate of the uniform partition is supposed to be $\delta=1$ (Definition 1);

- $\mathscr{U}$ is the finite alphabet of inputs (just after the Definition 1 ;

- $q:\left(x \in \mathscr{P}_{i}\right) \mapsto i$ is the output quantizer. Without loss of generality it is supposed to be the floor function, acting componentwise (just after the Definition 11;

- $\pi_{p}$ is the canonical projection on the first $p$ coordinates (Remark 1);

- $\lfloor\cdot\rfloor$ is the floor function, acting componentwise (just after Remark 1 ; ;

- $\left\langle v_{1}, \ldots, v_{i}\right\rangle$ denotes the linear subspace generated by the vectors $v_{1}, \ldots, v_{i} \in \mathbb{R}^{d}$ (Notations before Definition 4);

- $Q$ is quantization-diagonal set (Definition44;

- $X(k), U(k), F$ (capital letters) are used to indicate states, inputs and updating function of the doubled-system (3);

- $(\mathbb{X}, d)$ is a complete metric space used in the IFS setting (Definition 6);

- $\mathfrak{F}_{t}=\left\{\left\{F_{i_{1}} \circ \ldots \circ F_{i_{t}}\right\}: i_{1}, \ldots, i_{t} \in\{1, \ldots, n\}\right\}$ is the set of all possible concatenations of length $t$ of maps of an IFS (Definition 8 ;)

- $\mathscr{A}$ denotes the attractor of a system, of an IFS (Definition 9 ;

- $\mathscr{I}$ denotes the invariant set of an IFS (Definition 10;

- $\phi$ indicates the function that associates to each input sequence its limit point (Theorem 11;

- $G_{k}, I G_{k}, E G_{k}$ are respectively the graph of depth $k$ associated to the attractor $\mathscr{A}$, the internal invertibility graph, and the external invertibility graph (Definitions 12 . 13);

- $V_{I G_{k}}, V_{E G_{k}}$ denote respectively the set of vertices of the internal and external invertibility graph (Definition 13;

- $\partial$ indicates "topological boundary of ..." (Theorem 2);

- $\backslash$ denotes the set difference (Theorem 2];

- $Z(k), G_{U(k)}$ are used to indicate the state and the updating map of the doubledinverse system(4);

- $\mathscr{R}$ is the attractor of the doubled-inverse of a joint expansive system (Theorem 37;

- $\mathscr{V}=\mathscr{U}-\mathscr{U}=\left\{u-u^{\prime}: u \in \mathscr{U}, u^{\prime} \in \mathscr{U}\right\}$ is the input set of the difference set (Definition 16)

- $e_{i}$ is the $i$-th vector of the canonical basis of $\mathbb{R}^{d}$ (Notations before Definition 16);

- $\operatorname{frac}(\cdot)$ denotes the fractional part, acting componentwise (Notations before Definition 16];

- $\varpi_{i}: \mathbb{R}^{d} \rightarrow \mathbb{R}$ denotes the orthogonal projection on the $i$-th coordinate (Notations before Definition 16;

- $z(k), v(k)$ indicate respectively the state and the input of the difference system (Definition 16;

- $D_{k_{1}}^{k_{2}}\left(z(0), v(1), \ldots, v\left(k_{2}\right)\right)$ denotes the sequence $\left(\pi_{p} z\left(k_{1}\right), \ldots, \pi_{p} z\left(k_{2}\right)\right)$ generated by the difference system with initial condition $z(0)$ and inputs $v(1) \ldots, v\left(k_{2}\right)$ (Definition 177;

- $\mathbb{S}_{D}(B, \mathscr{U})$ denotes the set of matrices $A \in \mathbb{R}^{d \times d}$ such that the system 2 is ULDI (before Definition 23); 
- $\mathbb{S}(B, \mathscr{U})$ denote the set of matrices $A \in \mathbb{R}^{d \times d}$ such that the system 22 is ULI (before Definition 23);

- $I_{0}=\left\{\left(t_{1}, \ldots, t_{d}, t_{1}+s_{1}, \ldots, t_{p}+s_{p}, \tilde{t}_{p+1}, \ldots, \tilde{t}_{d}\right)^{T}: t_{i}, \tilde{t}_{j} \in \mathbb{R}, s_{k} \in\right]-1,1[\}$

- $d_{i}(X)$ is the measure of a distance defined in Definition 23 .

- $\Omega_{i}$ is the union of the two coordinate axes of $\left\langle e_{i}, e_{d+i}\right\rangle$ (Definition 23);

- $\mathbb{Q}\left[\zeta_{1}, \ldots, \zeta_{N}\right]$ is the ring of polynomial in the variables $\zeta_{1}, \ldots, \zeta_{N}$, with coefficients in $\mathbb{Q}$ (Definition 21);

- $Q^{\prime}$ denotes the "strip" $\left\{\left(\begin{array}{l}t \\ t\end{array}\right)+\left(\begin{array}{l}s \\ 0\end{array}\right): s \in\right]-1,1[, t \in \mathbb{R}\}$ (After the proof of Theorem 7);

\section{ACKNOWLEDGEMENTS}

This work has been partially supported by the European Commission under contract IST 224428 (2008) "CHAT - Control of Heterogeneous Automation systems: Technologies for scalability, reconfigurability and security", and contributes to the goals of CONET, the Cooperating Objects Network of Excellence, contract FP7-2007-2-224053.

\section{REFERENCES}

[1] Artin M, (1991) Algebra. Prentice-Hall

[2] Baker A, (1993) Trascendental number theory. Camb Univ Press

[3] Barnsley M, (1993) Fractals everywhere. Acad Press inc

[4] Bicchi A, Marigo A, Piccoli B, (1992) On the reachability of quantized control sytems. IEEE Trans Autom Control 47(4):546-563

[5] Bobylev N A, Emel'yanov S V, Korovin S K (2000) Attractor of discrete controlled systems in metric spaces. Comput Math Model 11(4):321-326

[6] Brockett R W, Mesarovic M D (1965) The reproducibility of multivariable control systems. J Math Anal Appl 11:548-563

[7] Carli R, Fagnani F, Speranzon A, Zampieri S (to appear) Communication constraints in the state agreement problem. Automatica

[8] Chitour Y, Piccoli B (2001) Controllability for discrete systems with a finite control set. Math Control Signal Syst 14:173193

[9] Delchamps D F (1990) Stabilizing a linear system with quantized state feedback. IEEE Trans Autom Control 35(8):916-924

[10] Dubbini N, Piccoli B, Bicchi A (2008) Left invertibility of discrete systems with finite inputs and quantized output. Proc 47th IEEE Conf Decis Control 4687-4692

[11] Edelmayer A, Bokor J, Szabó Z, Szigeti F (2004) Input reconstruction by means of system inversion: a geometric approach to fault detection and isolation in nonlinear systems. Int J Appl Math Comput Sci 14(2):189-199

[12] Falconer K, (2003) Fractal geometry, mathematical foundations and applications. John Wiley and Sons

[13] Gwozdz-Lukawska G, Jachymski J (2005) The Hutchinson-Barnsely theory for infinite interated function systems. Bull Aust Math Soc 72:441-454

[14] Inoue E, Ushio T, (2001) Chaos communication using unknown input observer. Electron Commun Jpn pt 3 84(12):21-27

[15] Hardy G H, Wright E M (1979) An introduction to the theory of numbers. Oxf Sci Publ

[16] Massey J L, Sain M K (1969) Invertibility of linear time-invariant dynamical systems. IEEE Trans Autom Control, AC-14(2)141-149

[17] Massey J L, Sain M K, (1968) Inverses of linear sequential circuits. IEEE Trans Computers C-17:330-337

[18] Morse A S, Wonham W M (1971) Status of noninteracting control. IEEE Trans Automat Control, 16(6):568-581

[19] Picasso B, Bicchi A, (2007) On the stabilization of linear systems under assigned I/O quantization. IEEE Trans Autom Control 52(10):1994-2000

[20] Respondek W (1990) Right and Left Invertibility of Nonlinear Control Systems. In: Sussmann (ed) Nonlinear Controllability and Optimal Control, NY pp 133-176

[21] Silverman L M (1969) Inversion of multivariable linear systems. IEEE Trans Automat Control 14(3):270276

[22] Sontag E D, (1998) Mathematical control theory: deterministic finite dimensional systems. Springer, NY

[23] Szanier M, Sideris A (1994) Feedback control of quantized constrained systems with applications to neuromorphic controller design. IEEE Trans Autom Control 39(7):1497-1502 
[24] Tatikonda S C, Mitter S (2004) Control under communication constraints. IEEE Trans Autom Control 49(7):1056-1068

[25] van Schuppen J H (2004) Decentralized control with communication between controllers. In: Blondel V D, Megretski A (ed) Princet Univ Press, Princeton, pp 144-150

[26] Vu L, Liberzon D (2006) Invertibility of switched linear systems. Proc 45th IEEE Conf Decis Control 4081-4086

Nevio Dubbini: Department of Mathematics “L. Tonelli”, University of Pisa, Pisa, Italy, TEL: ++390502213275, DUBBINI@MAIL.DM.UNIPI.IT

Benedetto Piccoli: Institute for the applications of CAlculus “M. Picone”, CNR, Rome, ITALY

Antonio Bicchi: Interdepartmental Center "E. Piaggio", University of Pisa, Pisa, Italy 